\title{
0 mouse, where art thou? The Mouse Position Surveillance System (MoPSS)—an RFID-based tracking system
}

\author{
Anne Habedank ${ }^{1}$ (D) $\cdot$ Birk Urmersbach $^{1} \cdot$ Pia Kahnau $^{1} \cdot$ Lars Lewejohann $^{1,2}$
}

Accepted: 29 March 2021 / Published online: 3 August 2021

(C) The Author(s) 2021

\begin{abstract}
Existing methods for analysis of home cage-based preference tests are either time-consuming, not suitable for group management, expensive, and/or based on proprietary equipment that is not freely available. To correct this, we developed an automated system for group-housed mice based on radio frequency identification: the Mouse Position Surveillance System (MoPSS). The system uses an Arduino microcontroller with compatible components; it is affordable and easy to rebuild for every laboratory because it uses free and open-source software and open-source hardware with the RFID readers as the only proprietary component. The MoPSS was validated using female C57BL/6J mice and manual video comparison. It proved to be accurate even for fast-moving mice (up to $100 \%$ accuracy after logical reconstruction), and is already implemented in several studies in our laboratory. Here, we provide the complete construction description as well as the validation data and the results of an example experiment. This tracking system will allow group-based preference testing with individually identified mice to be carried out in a convenient manner. This facilitation of preference tests creates the foundation for better housing conditions from the animals' perspective.
\end{abstract}

Keywords Behavior $\cdot$ Preference test $\cdot$ mice $\cdot$ Laboratory animals $\cdot$ Home cage $\cdot$ Group housing $\cdot$ Automated recording · Tracking $\cdot$ RFID $\cdot$ Refinement

\section{Introduction}

Preference tests are increasingly used to improve the housing and living conditions of laboratory animals. Such test procedures allow the animals' point of view to be directly involved in the refinement process. In order to get

Supplementary Material such as 3D printing templates, Arduino code, the $\mathrm{R}$ evaluation script, and raw data of the validation experiment can be found under: https://zenodo.org/record/4650404

If there is interest in the video recordings of the validation experiment, please contact us via e-mail.

Anne Habedank

anne.habedank@bfr.bund.de

Birk Urmersbach

birk.urmersbach@bfr.bund.de

1 German Center for the Protection of Laboratory Animals (Bf3R), German Federal Institute for Risk Assessment (BfR), Max-Dohrn-Str. 8-10, 10589 Berlin, Germany

2 Institute of Animal Welfare, Animal Behavior and Laboratory Animal Science, Freie Universität Berlin, Königsweg 67, 14163 Berlin, Germany a meaningful impression of the choices made, the tests should largely reflect normal laboratory conditions and allow to record the choice behavior without interference by an experimenter. This is at best realized using home cage-based preference tests (Habedank, Kahnau, Diederich, \& Lewejohann, 2018). For mice, the apparatus for such a choice test usually consists of two (Kawakami et al., 2012; Kirchner, Hackbarth, Stelzer, \& Tsai, 2012; Loo, Blom, Meijer, \& Baumans, 2005) or more (Ago, Gonda, Takechi, Takeuchi, \& Kawakami, 2002; de Weerd, Loo, Zutphen, Koolhaas, \& Baumans, 1997; Godbey, Gray, \& Jeffery, 2011) connected cages, directly connected via tubes or with a center cage. Animals are given continuous access to the options presented in each cage. In order to measure preference, either the nest position (Loo et al., 2005; Baumans, Schlingmann, Vonck, \& van Lith, 2002) or the compartment in which the animals spent more time (Blom et al., 1992; Freymann, Tsai, Stelzer, \& Hackbarth, 2015, 2017; Godbey et al., 2011; Kawakami et al., 2012; Kirchner et al., 2012) is then monitored and regarded as the favored one (Habedank et al., 2018).

Thus, home cage-based preference tests are based on binary or multiple choices, and they are designed to rank 
preferences, not to assess the strength of preference or the "demand" for this resource (Kirkden \& Pajor, 2006). In this manner, the preference of mice was already investigated regarding bedding material (Blom, Tintelen, Vorstenbosch, Baumans, \& Beynen, 1996; Kirchner et al., 2012), the provided amount of it (Freymann et al., 2015, 2017), nesting material (Ago et al., 2002; de Weerd et al., 1997), shelters (Loo et al., 2005), cage change interval (Godbey et al., 2011), ventilation (Baumans et al., 2002; Krohn \& Hansen, 2010), temperature (Gaskill, Rohr, Pajor, Lucas, \& Garner, 2009, 2011; Gaskill et al., 2012) and environment (Kawakami et al., 2012). Further husbandry conditions, which to our knowledge are not yet fully investigated in this manner are, e.g., brightness, humidity, and different items of enrichment such as structural elements or equipment for active engagement.

When conducting a home cage-based preference test, it can be distinguished between the active (dark) and the inactive (light) phase to analyze the data (Freymann et al., 2015; Lewejohann \& Sachser, 2000). This is especially important if the tested cage conditions are predominantly associated with active (e.g., running wheel) or inactive behavior (e.g., nesting material). Social species of laboratory animals such as mice are usually kept in groups. Social conditions are likely to influence the choice of individual mice; for example the sleeping temperature might be influenced by the presence of other animals (Gordon, Becker, \& Becker, 1998). Thus, generally speaking, animals that are living in groups under normal laboratory conditions should also be tested in groups. However, measuring the preference of a group of mice is a far greater challenge than measuring singly housed mice, and thus, many of the preference studies investigated individual mice instead of groups (Blom et al., 1992, 1996; de Weerd et al., 1997; Kawakami et al., 2007, 2012). When testing groups (Freymann et al., 2015, 2017; Godbey et al., 2011; Gaskill et al., 2009, 2011, 2012; Kirchner et al., 2012), individuals in one group can influence each other (Loo, de Groot, Zutphen, \& Baumans, 2001; Shemesh et al., 2013; Valsecchi \& Galef, 1989), so that the results from one group might have to be counted as a single unit. More recent advances in statistical methods allow including "group" as a random factor in the model, but still the total number of animals might have to be increased to account for such group effects.

Of the available methods to analyze a home cagebased preference test, most do not carry the capability to sufficiently cope with implicit challenges of choice tests. For example, monitoring only the nest position (Baumans et al., 2002; Loo et al., 2005) causes little costs with regard to equipment and time, but provides mainly information on where the mice spent their inactive time and thus does not reflect temporal distribution of individual preferences. The most common analysis of home cage-based preference tests is therefore done by video recordings (Ago et al., 2002; Gaskill et al., 2009, 2011; Godbey et al., 2011; Kawakami et al., 2007). However, video analysis is very timeconsuming, especially when it is necessary to distinguish between individuals. For this reason, some research groups only analyze part of the recordings instead of a continuous tracking (every 5 min: Kawakami et al., 2007; every $10 \mathrm{~min}$ : Gaskill et al., 2009, 2011, 2012; every 60 min: Godbey et al., 2011), whereby the time saving is at the expense of the accuracy of the measurement. Analysis of the videos in a more automated manner by using video tracking software (Nath et al., 2019; Noldus, Spink, \& Tegelenbosch, 2001; Rao et al., 2019) is by now not advanced enough to ensure decent tracking of individual mice in the husbandry cage.

However, there are other techniques which allow automated tracking: For example, in the connecting tunnels, light barriers can be implemented to record whenever an animal changes cages (Blom et al., 1992, 1996). This method allows easy continuous tracking without much analysis effort. However, this approach is not suitable for group housing because aside from lacking individual detection, the determination of direction of passages is erroneous if sensors can be triggered by more than one animal. Similar problems would also arise if using digital scales below the cages combined with an automated tracking program (Krohn \& Hansen, 2010).

To combine automated and individual detection, telemetry can be used by either implanting a rather large, batterypowered transponder (Kawakami et al., 2012) or injecting a smaller, passive transponder for radio-frequency identification (RFID) (Freymann et al., 2015, 2017; Kirchner et al., 2012). The latter method is also very commonly used not just for choice tests but to record general patterns of mice (Bains et al., 2016; de Chaumont et al., 2019; Freund et al., 2013; Weissbrod et al., 2013), rats (Redfern et al., 2017) and birds (Bridge et al., 2019).

All in all, there have been several systems described which automatically track the position of mice. However, these systems are often based on proprietary equipment, only commercially available and expensive (Actual Home Cage Analyzer by Actual Analytics and AstraZeneca: Bains et al., 2016; Redfern et al., 2017; a sorting system by PhenoSys: Winter \& Schaefers, 2011; PhenoWorld and other TSE products: Castelhano-Carlos, Costa, Russig, \& Sousa, 2014; Linnenbrink \& von Merten, 2017). In addition, most of these systems are not designed for preference tests, and thus would need reconfiguration to meet the demands of home cage-based preference tests. This is also the case for tracking software like the closed-source software EthoVision (Noldus et al., 2001) or the nonproprietary software MAPS (Endo et al., 2018), AnimApp (Rao et al., 2019), DeepLabCut (Nath et al., 2019), and 
MiceProfiler (de Chaumont et al., 2012), which are not set to track mice in a common husbandry cage with a grid top and optional enrichment. There is further development of the MiceProfiler combined with RFID; however, for this method, two transponders have to be implanted, which is a disadvantage (Weissbrod et al., 2013). Another system, the Mouse Tracker (de Chaumont et al., 2019), uses only one RFID transponder but also does not work in a common husbandry cage. One promising approach is a system that was actually developed for home cage-based preference tests, called the DoubleCage (Tsai, Nagelschmidt, Kirchner, Stelzer, \& Hackbarth, 2012). However, this system is also based on proprietary equipment, not freely available and has limited accuracy. Another approach is a study conducted with birds, but they use non-implantable transponders and is geared to detect animal species moving slower than mice (Bridge et al., 2019). Thus, for a home cage-based preference test with group-housed mice, a reliable, lowcost, adaptable, and time efficient analysis method is still missing. (An overview of the described methods so far and their advantages and disadvantages is summarized in Table 1.)

For this reason, we developed an automated system based on RFID that is affordable for everyone (all in all $<150$ euros), not based on proprietary software or equipment (except for the RFID readers), easy to (re)build, and suitable for individual tracking in group-housed mice: the Mouse Position Surveillance System (MoPSS). It consists of an Arduino MKR WIFI 1010 microcontroller and two RFID controllers with two antennas (with the RFID controllers as the only proprietary hardware we used). In order to read an RFID signal, the transponder has to stay within the electromagnetic field of the antenna for around $30 \mathrm{~ms}$. Mice are capable of very fast movements, and can reach up to $18.0 \mathrm{~m} / \mathrm{min}$ without training on a treadmill (Billat, Mouisel, Roblot, \& Melki, 2005), 23-31.8 m/min after training (Hollinski et al., 2018), $67 \mathrm{~m} / \mathrm{min}$ on a running wheel (Bono, Adlam, Paterson, \& Channon, 2006) and possibly even higher velocities during short sprints and jumping. Therefore, additional barriers were added in the connecting tube between the cages in order to slow down the movements in the vicinity of the antennas. Here, we provide the experimental validation of the system with a group of 7-week-old female C57BL/6J mice as well as the complete implementation description: To facilitate the rebuilding of the MoPSS in other laboratories, we supply the construction plan, the Arduino code, and the 3D print design of the barriers. We also describe an additional analysis method for the data which uses logical reconstruction to further improve the obtained data. With the help of this paper, the MoPSS can be rebuilt by any laboratory and/or altered with regard to example, other species).

\section{The Mouse Position Surveillance System (MoPSS)}

\section{General principle}

The basic experimental setup consists of two cages that are connected by a Perspex tube (40 $\mathrm{mm}$ in diameter) passing two RFID antennas (see Fig. 1). As the system relies on RFID, all animals need to have an RFID transponder implanted. We recommend placing it under the skin in the neck region. For best reading performance, the transponder must be implanted lengthwise (rostrocaudal). When a mouse moves through the tube and enters the magnetic field emitted by the RFID antenna, the transponder is read and the transponder number, antenna number, and current timestamp are saved onto a microSD card (32 GB). For the analysis, a mouse detected at the left RFID antenna is counted as being in the left cage, and a mouse detected by the right RFID antenna is counted as being in the right cage. It is possible to subtract the transition duration so as to not add it to one of the cages. However, as mice usually pass very quickly through the tube, we argue that the passage time is neglectable. The main challenge while developing the apparatus was that the mice were too fast for the RFID detectors, i.e., they spent less time than necessary within the read range during the read cycle. In addition, if multiple mice were in the range of the same antenna, interference led to poorer detection as well. Therefore, we added two barriers inside the connecting tube, each obstructing approximately $40 \%$ of the tubes' diameter and thereby forcing the mice to slow down in the vicinity of the antennas while passing the barriers.

\section{Electronics}

The MoPSS system consists of an Arduino MKR WiFi 1010 microcontroller with an attached Arduino MKR SD PROTO SHIELD holding a microSD card (Samsung, South Korea) for data collection and control of the RFID reader modules. A small lithium-polymer battery is attached to the Arduino with a 3D-printed mount (Supplement File: MoPSS_Battery_Holder.stl) including a dedicated switch integrated in the housing, to allow disconnecting the battery.

Two RFID reader modules (RFIDRW-E-TTL, Priority 1 Design, Australia) and two external antennas (RFIDCOIL49A, Priority 1 Design, Australia) are used for reading the RFID signals. In order to protect the antenna coils, a support that fitted exactly around the Plexiglas tubes was used, first premade and later self-built using a 3D printer (files available in the Supplement).

The mainboard for the MoPSS system is built on a perfboard and provides the connections between the 


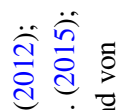

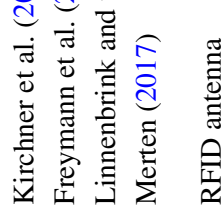

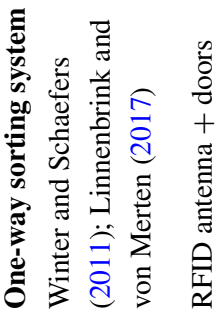

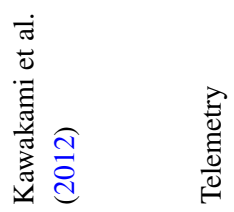

蔗高

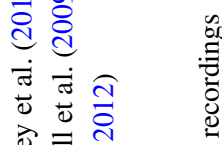

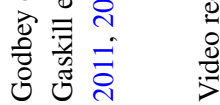

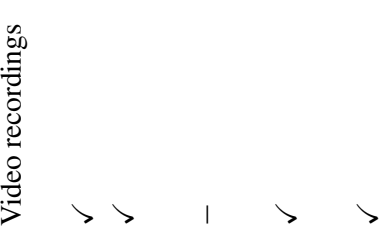

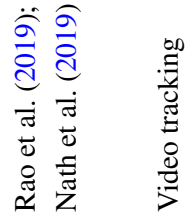

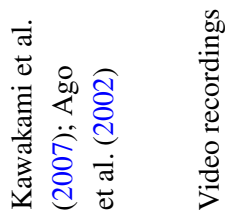

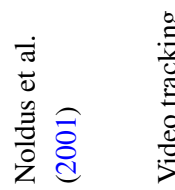

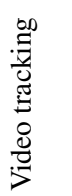

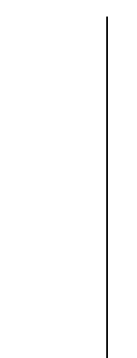

हี

远

.0

它

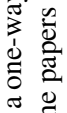

ส

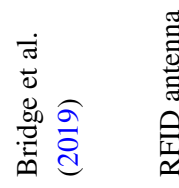

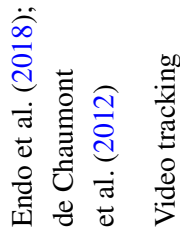

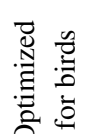

跑

焉

崩

$\stackrel{0}{0}$

迎 है

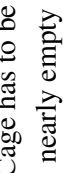

言管

空

:

$\tilde{\omega}$

.

离

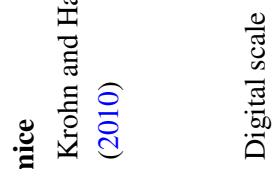

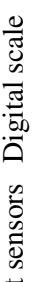

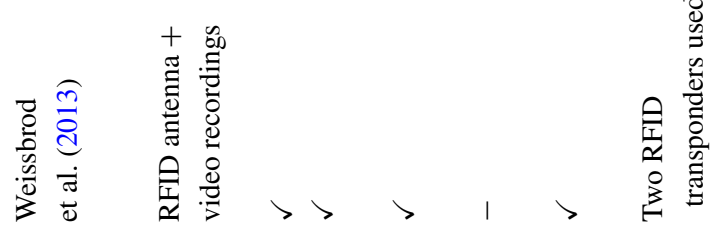

$\vec{\otimes}$
के
iे

造

(5)

छั

可

䒕

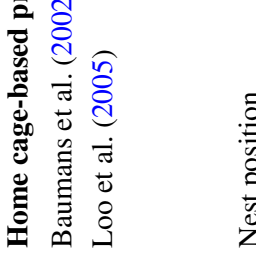

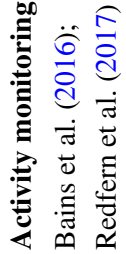

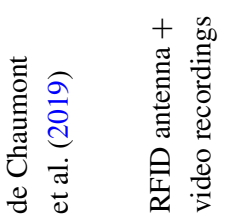

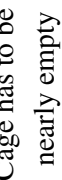

离

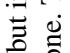

.

ह

令哭

它

总

:

혼 을

究

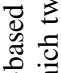

离 $\frac{9}{3}$

政考

:

ฮี

है

के

总

훨

言

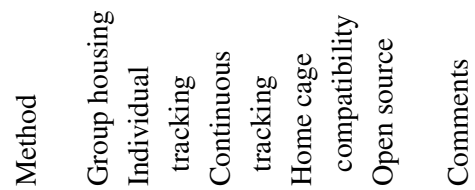

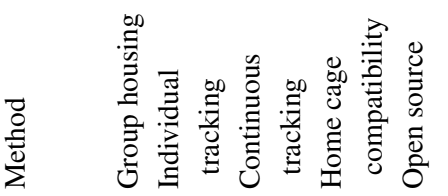

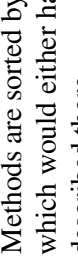




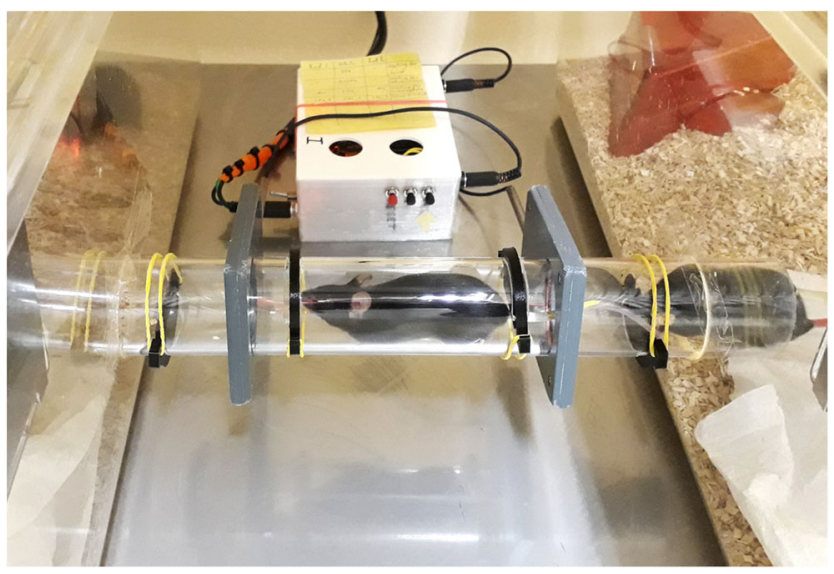

Fig. 1 Setup of a home cage-based preference test using the MoPSS. Two cages are connected via a tube with four barriers and two RFID antennas

Arduino and the RFID modules. Three LEDs for visual feedback, and three push buttons for user input and reset are added. The mainboard also provides pin header connections for the push buttons, antenna barrel connectors, and the power connector (Fig. 2).

The box for the MoPSS system is printed using polylactic acid (PLA) and consists of a bottom unit with a cutout for easy access to the microSD card and mounting holes for the buttons, etc. A lid with venting holes for the box is also included (Supplement File: MoPSS_Case.stl and MoPSS_Lid.stl).

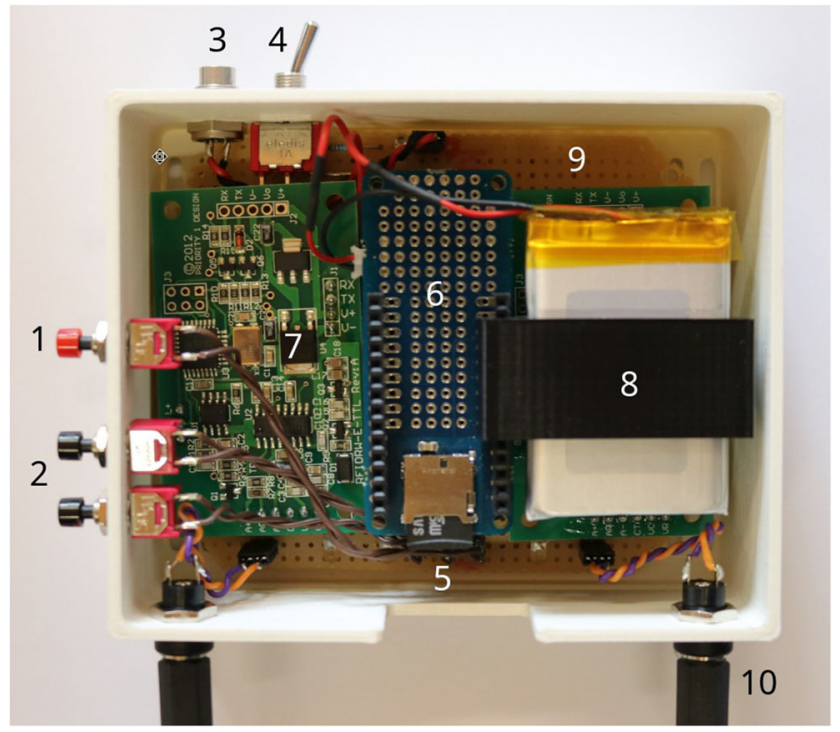

Fig. 2 Inner workings of the MoPSS: 1 reset button, 2 button B1 and B2 for user input, 3 power connector, 4 battery on/off switch, 5 microSD card, 6 MKR SD SHIELD, Arduino below, 7 RFID reader module, 8 lithium-polymer battery with holder, 9 mainboard, 10 antenna connector

\section{Barrier construction}

Barriers were implemented to slow the mice down while moving through the 31-cm-long tube (diameter: $4 \mathrm{~cm}$ ). To achieve this, we applied four barriers: For both RFID antennas, a barrier from below $(5 \mathrm{~cm}$ from the end of the tube) and a barrier from above $(10 \mathrm{~cm}$ from the end of the tube) are inserted (see Fig. 3). To install the barriers, 5mm-wide slits have to be cut into the tube. Barriers block about $40 \%$ of the tubes' diameter and are $4 \mathrm{~mm}$ wide. The barriers are made with a 3D printer (Ultimaker 3 Extended, Ultimaker B.V., The Netherlands) using Ultimaker black PLA as material. They are designed with two hooks on either side, so they can be easily inserted into the tube and fixed with a rubber band. The barrier template for the 3D printer is offered (Supplement File: Barrier.stl). In addition, to facilitate the cutting of the tube, a 3D template is provided (Supplement File: Gauge_Tunnel_Barriers.stl), which assists in drawing exact cutting lines onto the tube.

\section{Transponders}

We use transponders according to ISO 11784/85 (FDXB transponders, Euro I.D., Germany). The transponder needs to be implanted rostrocaudal for optimal detection sensitivity. The best read performance is achieved when the RFID transponder is oriented lengthwise $\left(0^{\circ} / 180^{\circ}\right)$ to the antenna where read ranges of approximately $4 \mathrm{~cm}$ can be achieved. If a transponder were oriented transversely $\left(90^{\circ} / 270^{\circ}\right)$ to the antenna, the read range would approach $0 \mathrm{~cm}$. For more details on the transponder implantation procedure, see section Experiment 1, Animals.

\section{Software}

The Arduino and RFID reader modules each run different software. The RFID modules use proprietary software while the software for the Arduino is available in the Supplement.

RFID modules The RFID modules are connected to an antenna each in order to read the unique number of the RFID tag that is within read range and transmit this tag number to the Arduino.

As soon as an RFID tag enters the read range of the antenna, the tag number is read by the RFID module and transmitted to the Arduino. However, the tag number is only transmitted when the tag newly enters the read range.

In order to eliminate interference between the two RFID antennas in close proximity, we decided to enable only one RFID reader at a time for $100 \mathrm{~ms}$, alternately switching between both. As a consequence, every time an RFID reader is re-enabled, any tag it reads will be automatically transmitted because the tag appears as "new" to the RFID 


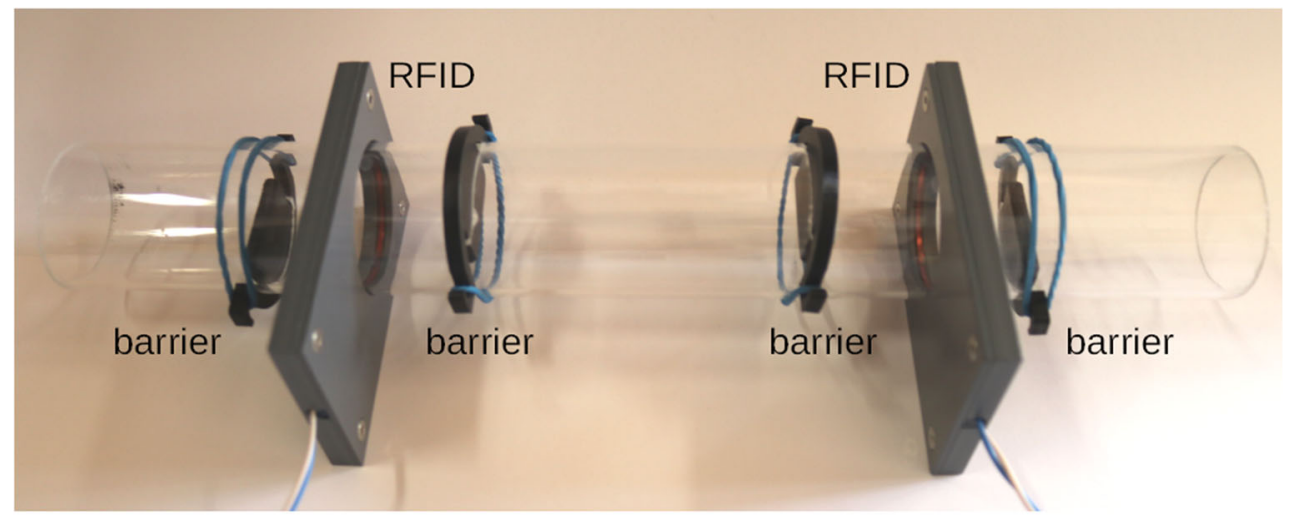

(a)

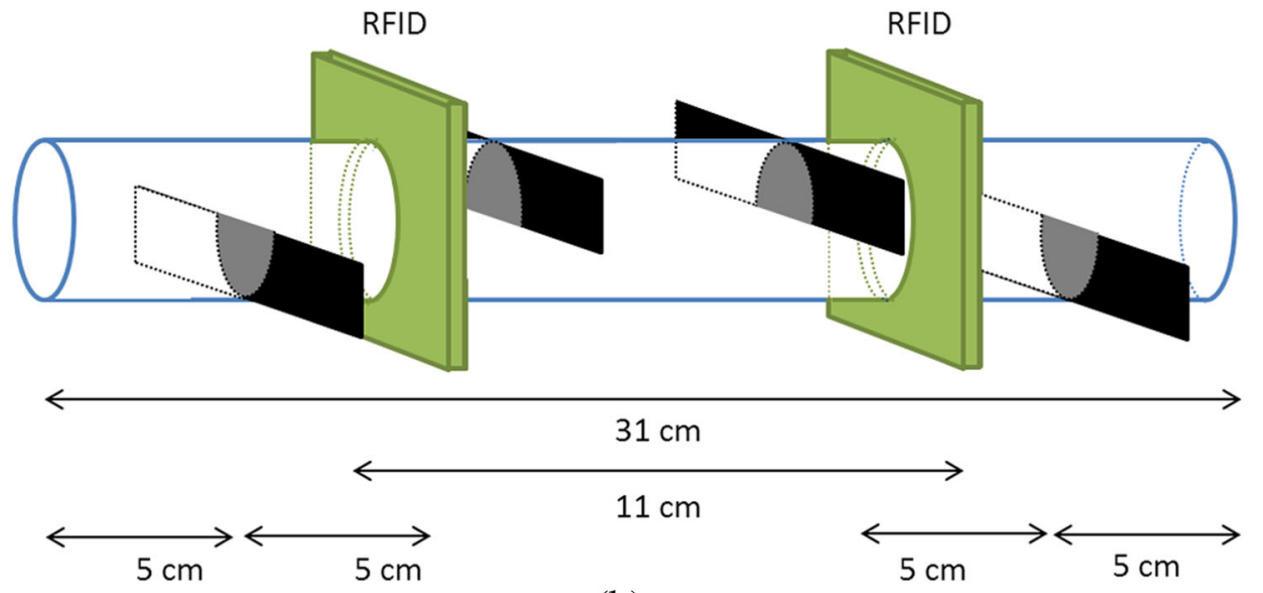

(b)

Fig. 3 Picture of barrier construction (a) and schematic drawing (b) of barrier construction. RFID RFID antennas, black barriers

reader. This enables us to easily detect when an RFID tag is no longer within the read range of the reader.

Arduino The Arduino is handling the processing of the RFID tag numbers that are communicated by the RFID modules and adds additional functionality such as visual feedback and logging. Additionally, the Arduino controls charging of the battery that allows coping with short-term power loss.

During startup, the Arduino connects via Wi-Fi to the Internet in order to update the internal real time cock, which is then used during logging to provide accurate timestamps for all RFID tag detections. For the timestamps, the Unix time is used, which is easily processed in further analysis and indifferent to time zones. After successful synchronization, the Wi-Fi on the Arduino is no longer required and turned off, thereby greatly reducing power consumption. The battery allows independent operation of the Arduino, guarding the system in case of external power loss for roughly $26 \mathrm{~h}$. Even though RFID capability is lost while running on battery power, the reader modules will restart without adverse consequences once power is restored. Battery power can also be used for the startup of the MoPSS system at a different location, for example, if there is no Wi-Fi available inside the animal facility.

The Arduino also controls the LEDs on the mainboard communicating the different states between power on and ready for operation. At the time of writing these are: "searching for Wi-Fi network", "fetching time from network time protocol server;", "ready for operation", and "error during setup" indicating a faulty/missing microSD card, inability to connect to the network/synchronize the time. During operation, two red LEDs corresponding to the two RFID reader modules are also used to indicate the detection of a tag.

In the event of a successful RFID tag detection, the Arduino saves the data to the microSD card: the antenna number by which the tag was read (A1/A2), the current time (e.g., 1567081062), the tag number (e.g., 900_200000123456) and a flag (E) indicating that this detection corresponds to a mouse entering the read range. When the transponder is no longer detectable, an additional 
Table 2 Example of the recorded data provided by the MoPSS

\begin{tabular}{llll}
\hline Antenna no. & Unix time & Tag number & Entry/EXit flag \\
\hline A1 & 1567081062 & $900 \_200000123456$ & E \\
A1 & 1567081063 & $900 \_200000123456$ & X \\
A2 & 1567081071 & $900 \_200000123456$ & E \\
A2 & 1567081072 & $900 \_200000123456$ & X \\
\hline
\end{tabular}

entry is made containing the antenna number, current time, the tag number and the flag $\mathrm{X}$ to indicate an exit from the read range. See Table 2 for an example.

\section{Data evaluation}

Although accuracy of the RFID detections was very high (see section Experiment 1 Validation, Results), there were still a few missed detections. We therefore conducted an in-depth analysis of the possible combinations of missed detections with the known detections to identify cage changes despite missing data. The resulting $\mathrm{R}$ script can systematically analyze raw data and reliably reconstruct cage changes in the few cases of missing detections. The complete description of this procedure can be found in the Supplementary Material.

\section{Experiment 1: Validation}

In order to compare the accuracy of the MoPSS to manual video analysis, we performed a validation experiment using both methods in parallel.

\section{General procedure}

A group of 12 young mice was habituated for 6 days to the MoPSS, including the barrier system in the connection tube before a 24-h video recording was performed. The video recording was then analyzed with regard to cage changes, and these were compared to the cage changes detected by the MoPSS.

\section{Animals}

We chose $\mathrm{C} 57 \mathrm{BL} / 6 \mathrm{~J} \mathrm{CrL}$ mice because this is the most commonly used mouse strain. Twelve female C57BL/6J $\mathrm{CrL}$ mice, kept as one group, were used for this experiment. They were purchased in June 2019 at the age of 4 weeks from a commercial breeder (Charles River, Sulzfeld, Germany) and had different mothers and had different nurses to prevent any breeding-related effects. At 5 weeks of age, transponders (FDX-B transponder according to ISO $11784 / 85$, Euro I.D., Germany) were implanted

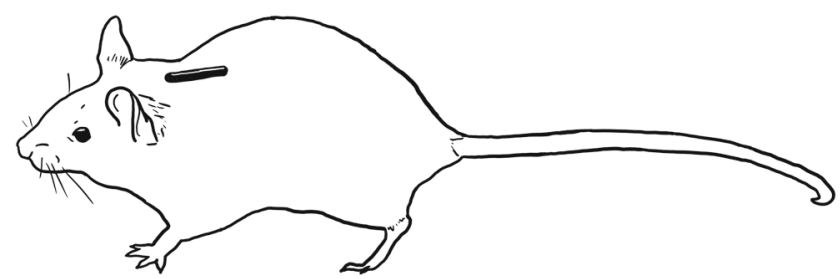

Fig. 4 Schematic drawing transponder position. (CAnne Habedank

subcutaneously in the neck region (see Fig. 4). In order to prevent potential harm inflicted by the implantation procedure, the mice obtained an analgesic (Meloxicam) the evening before implantation. The transponder injection itself was performed under anesthesia (Isoflurane) and the RFID transponder was injected directly behind the ears subcutaneously in the neck, so that it was oriented rostrocaudal. After transponder injection, the mice were placed in a separate cage with bedding and paper for monitoring until they were fully awake again. They were then returned to their home cage.

\section{Housing}

In the first weeks, the mice were kept in a type IV Makrolon cage $(\mathrm{L} \times \mathrm{W} \times \mathrm{H}: 598 \times 380 \times 200 \mathrm{~mm}$, Tecniplast, Italy) with a filter top. Food (autoclaved pellet diet, LAS QCDiet, Rod 16, Lasvendi, Germany) and tap water (two bottles) were available ad libitum. The cage was equipped with bedding material (Poplar Granulate 2-3 mm, Altromin, Germany) of 3-4 cm height, two red houses (The MouseHouse, Tecniplast), four papers, four cotton rolls, 12 strands of additional paper nesting material, and four wooden bars to chew on. The cage also contained a Perspex tube (40 $\mathrm{mm}$ in diameter, $17 \mathrm{~cm}$ long), which was used for tube handling (Hurst \& West, 2010; Gouveia \& Hurst, 2013).

For the validation of the MoPSS, when the mice were 6 weeks of age, they were moved into two type III Makrolon cages $(\mathrm{L} \times \mathrm{W} \times \mathrm{H}: 425 \times 276 \times 153 \mathrm{~mm}$, Tecniplast, Italy) with filter tops connected via a Perspex tube $(40 \mathrm{~mm}$ in diameter, $30 \mathrm{~cm}$ long) containing barriers from above and below (blocking $40 \%$ of the tube diameter with a thickness of $4 \mathrm{~mm}$; see description of barriers in the "Barrier construction"). The equipment described above for the type IV cage was equally split unto the two type III cages, except that only one cage contained the handling tube.

Room temperature was maintained at $22 \pm 3{ }^{\circ} \mathrm{C}$ and the humidity at $55 \pm 15 \%$. Animals were kept at a $12 \mathrm{~h} / 12 \mathrm{~h}$ dark/light cycle with the light phase starting at 8:00 a.m. (summer time). Between 7:30 and 8:00 a.m., a sunrise was simulated using a Wake-up light (HF3510, Philips, Germany). Once per week, the home cage system was cleaned and all mice were scored and weighed. In this 
context, the mice also received a color code on their tails (using an edding 750 paint markers) to facilitate individual recognition during video recording.

\section{Procedure}

With 6 weeks of age, the 12 female C57BL/6J mice were transferred into the test system, consisting of two cages connected with a tube containing four barriers and two RFID antennas (for details see "Housing" and "Barrier construction"). After 6 days of habituation to this setup, video recordings of the tube were made for $24 \mathrm{~h}$. To ensure continuous recording of mouse movement, we installed a red light source, which was automatically switched on during the dark phase. The video recordings were conducted with a webcam (Logitech C390e, Switzerland) using the recording software iSpy 64 (version 7.0.3.0), which automatically cut the videos into blocks of 1-h duration. The webcam was positioned in a way that ensured a clear view of the connecting tube and the MoPSS, which signaled every RFID detection via two separate red LEDs.

Afterwards, we collected the recorded data from the MoPSS and compared the detected cage changes with the 24-h video recordings: We fast-forwarded the video recordings until a mouse was visible and, slowing down the video, then monitored whether the MoPSS signaled via a blinking LED that the RFID tag number of the mouse was detected. In some cases, more than one mouse passed through the tube and an additional evaluation whether or not all mice were detected was conducted: The recorded data from the MoPPS were examined to verify that all RFID tag numbers were recorded at the corresponding timestamp. All missing detections were noted.

As described in "Data evaluation", in addition to just using the data as it was saved by the MoPSS, we also developed a method to improve the received data by means of logical reconstruction (searching the recorded data for inconsistencies in the order of cage changes; for details see "Data evaluation" and the Supplements). In the process of evaluating the $\mathrm{R}$ script for this logical reconstruction, parts of the video recordings were watched again to compare the results of the script against the true events.

\section{Results}

During the $24 \mathrm{~h}, 7382$ detections were recorded, including 2804 cage changes. On average, there are more than twice as many detections as cage changes because mice do not always change cages but sometimes also just stick their nose inside the RFID antenna (poke) and then return to the cage they came from. After a manual comparison of the recorded detections with the 24-h video recordings, we found nine missed detections, meaning an event in which one of two antennas did not detect the mouse (situation B and C from section Data Evaluation, Supplements). This led to an error rate of $0.122 \%$ of all the cage changes. There was no cage change detected on video for which both antennas did not detect the mouse (situation D from section Data Evaluation, Supplements), which would have not been possible to reconstruct due to the missing timestamps.

After analyzing the data by means of logical reconstruction (as described in section Data Evaluation, Supplements), we were able to infer the nine missing detections automatically and correct the corresponding cage changes. In this manner, the error rate was reduced to $0 \%$.

Analyzing the detections, we found that dwelling time between the readers was on average $1736 \mathrm{~ms} \pm 8255 \mathrm{~ms}$, with $87.33 \%$ of cage changes taking $\leq 3$ s and $94.27 \%$ taking $\leq 5 \mathrm{~s}$.

\section{Discussion}

Validating the MoPSS' detection with manual video analysis, we confirmed that the MoPSS reaches a very high accuracy. After logical reconstruction, the MoPSS detection matches $100 \%$ with the results of the manual video analysis. The only divergence arises in the timestamps-when one of the two antennas missed the passage, the timestamp of the second antenna had to be taken (as explained in section Data evaluation, Supplements). However, we can assume that the mouse was missed by the antenna only because it moved too fast out of the antenna's read range (about $5 \mathrm{~cm}$ before and behind the antenna). Thus, we argue that the missing timestamp and the timestamp from the second antenna should be differing only by a few seconds from the correct time, and it is reasonable to use it to replace the missing timestamp.

Note that the error rates reported above are only results of one group of mice, and thus they might not be representative for other groups, especially when differing in age, strain, or sex. Still, we regard the chosen test group as the optimal one for its purpose: The main difficulty, as explained above, was the velocity of the mice, and that is why we used very young and thus fast animals. The mice had 6 days of habituation to adjust to the new barrier setup. However, it is possible that the mice were not at their highest possible speed. In the study by Bono et al. (2006), it is described that maximum continuous speed increased until day 17 of training for female C57BL/6J mice (10 to 11 weeks old). Hollinski et al. (2018) described an increase in maximum continuous speed up until week 8 of training. Nevertheless, these studies were conducted on running wheels, whereas for our experiment the maximum speed over a distance of approximately $8 \mathrm{~cm}$ in a straight line is the most relevant, as this is the range of the RFID antenna. 
We believe our manual video analysis can be considered nearly flawless because, when in doubt, videos were played backwards or in slow motion. This also emphasizes the improvement the MoPSS is going to make, as an accurate analysis by video was very time-consuming.

Comparing the MoPSS' accuracy to the other available methods for home cage-based preference tests (which were described in the Introduction) proves difficult. First, accuracy can only be compared to manual analysis, which would make video recordings automatically the most accurate method. However, as we experienced during the development of MoPSS prototypes, especially when using group-housed mice, even manual analysis can be complicated. When mice climbed over each other, they were sometimes not distinguishable without the information provided by the RFID antennas.

Second, comparing the MoPSS' accuracy to other automated tracking systems is in some cases not possible because the studies do not provide any information on accuracy (Krohn \& Hansen, 2010; Linnenbrink \& von Merten, 2017) or any details on the tracking system except that they used one (Kawakami et al., 2012). We, on the other hand, reported very detailed how the accuracy was measured.

Third, of the remaining two automated tracking systems, the one described by Blom et al. (1992) only uses individually housed mice, which makes data acquisition far easier, but with the disadvantage that the transferability of gained results for group-housed mice remains questionable. In addition, Blom et al. (1992) and Tsai et al. (2012) use a correlation between relative dwelling times per cage based either on visual observations or automatically registered cage changes. This, however, does not provide general information on the error rate of the system; it merely states that there is no significant difference between the results. This, however, would change if a cage change was missed after the mice had stayed in this cage for several hours. The paper by Tsai et al. (2012) offers an error rate with $0.26 \%$ of misreported cage changes. In comparison, the MoPSS has an initial error rate (before logical reconstruction which corresponds to RFID reader accuracy) of $0.122 \%$ for missed detections. As explained in the section "Data evaluation", missed detections do not have to lead to a missed cage change if the first RFID antenna the animal was passing through was the one with the missed detection because only the second RFID antenna reports an actual change in position.

Fourth, it has to be noted that currently no automated tracking system can reach $100 \%$ accuracy at all times (without additional analysis of the data afterwards) because at this time, there are situations which cannot be identified by automated systems. For example, when a mouse passes through an antenna and another mouse passes the antenna at the same time, two RFID transponders are within the detection range and one RFID tag may obscure the other. However, this is a very rare scenario. Overall, we demonstrated that the MoPPS is equally accurate as video observation and much superior with regard to time taken for analysis.

\section{Experiment 2: Example data}

\section{General procedure}

Experiment 2 is an example of a home cage-based preference test conducted with the MoPSS. Please note that this preference test was performed to show that the MoPSS has the capability of tracking even a group of 12 mice easily. It is not our recommendation to conduct preference tests in such large groups, and because of that, the result of this experiment should not be generalized (see also Discussion). Two types of bedding material were compared, using one group of 12 mice. The preference test was performed in two consecutive rounds of 3 days each. Between rounds, the presentation side of the bedding materials was changed, starting the new round with freshly cleaned cages. The MoPSS was active during the whole duration of the experiment; however, only the second day of both rounds was used for analysis, providing the first day for habituation.

\section{Hypothesis}

We conducted a home cage-based preference test comparing two bedding materials: Pure (cellulose, JRS) and Comfort White (cellulose, JRS). Both bedding materials were known to the mice because they were used before in a conditioned place preference test as the conditioned stimuli. In this test, mice had shown a significant preference for Comfort White bedding during the 10-min habituation as well as during the final test after conditioning. Now, we wanted to investigate whether this preference would persist if mice had not only 10 min but several days of continuous access to the bedding materials.

\section{Animals}

Another group of 12 female C57BL/6J CrL mice was used for this experiment. This group was purchased in December 2017 at the age of 3 weeks from Charles River, Sulzfeld. The mice were born to different mothers and had different nurses in order to cope for any possible effects on behavior related to the prenatal and early postnatal phase within the inbred strain. With about 5 weeks, transponders (FDX-B transponder according to ISO 11784/85, Planet-ID, Germany) were implanted under the skin in the neck. The procedure was the same as for the group in Experiment 1, 
except that Meloxicam was given $2 \mathrm{~h}$ before the procedure instead of the previous evening. In addition, for two mice, the transponder implantation had to be repeated at the age of 8 weeks because they lost their transponder immediately after the first implantation.

This group of mice took part in multiple testing of prototypes to develop an automated tracking system. By the time the home cage-based preference test was performed to gain example data with the MoPSS, they were around 19 months old. In between, mice had also participated in other experiments, e.g., T-maze preference tests and conditioned place preference tests (the latter were pre-registered at the Animal Study Registry: Lewejohann Lewejohann, 2019a, b the former took place before the launch of the Animal Study Registry).

\section{Housing}

Outside experiments, mice were kept in two type IV Makrolon cages $(\mathrm{L} \times \mathrm{W} \times \mathrm{H}: 425 \times 276 \times 153 \mathrm{~mm}$, Tecniplast, Italy) with filter tops connected with a Perspex tube (40 $\mathrm{mm}$ in diameter), which was equipped in the same way as the two type III cages described for the group in Experiment 1.

\section{Procedure}

Because this group of mice was usually kept in a home cage system with two connected cages, those cages were identically equipped as always, except that we changed the normal bedding material for different ones: One cage was filled with Pure bedding (cellulose, Arbocel pure, JRS, J. Rettenmaier \& Söhne GmbH + Co KG, Germany) and one with Comfort White bedding (cellulose, Arbocel comfort white, JRS, J. Rettenmaier \& Söhne GmbH + Co KG, Germany) up to the same height of $3 \mathrm{~cm}$. Both beddings consisted of cellulose, while the usual bedding consisted of conifer wood (spruce/fir). For a picture of the different bedding materials, see Fig. 5. The connecting tube was similarly designed as described in Experiment 1, however, we only added barriers from below to facilitate their passing through the tube. This group was older, and one mouse was unusually hesitant towards new objects, which had already been observed during several other experiments, and we did not want to exclude it.

As it is possible that the spatial position in the room (and its light, noise, room air conditions) influences the preference of the mice (Blom et al., 1992), we performed two rounds, between which the presentation sides of the bedding materials were changed. This ensures a discrimination between side and bedding preference. The experiment lasted 7 days, with 3 days presenting bedding material Pure left and Comfort White right (round 1), then switching sides and presenting Pure right and Comfort White left to control for a spatial bias (round 2). On the first day of each round, the mice were placed into freshly cleaned and newly equipped cages, placing individual mice alternately into the left and right cage, dependent on the order they entered the handling tube. The first day was considered as a habituation day to get the mice accustomed to the new bedding material. The second day was then used for actual data recording. The third day was added for organizational reasons: After approximately $23 \mathrm{~h}$ of the third day, the mice were then taken out of the test setup and placed into a separate cage (which contained the spruce/fir bedding they usually had), while preparing the new setup. Mice were then placed into a freshly cleaned and newly equipped cage, this time with changed presentation sides of the bedding. Only the food was maintained; pellets of both cages were mixed and split for the two new cages. The tube connecting the cages as well as the barriers were not cleaned in between. In the second round (just as in the first

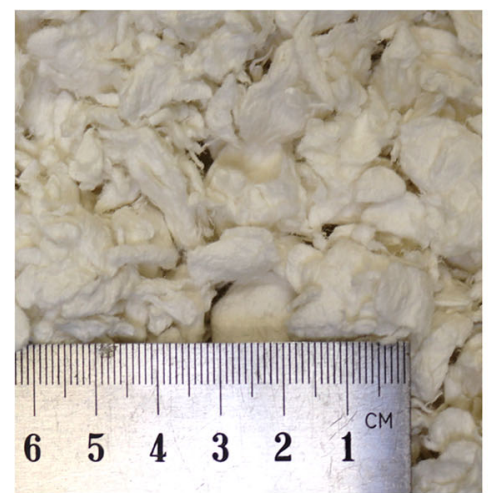

(a)

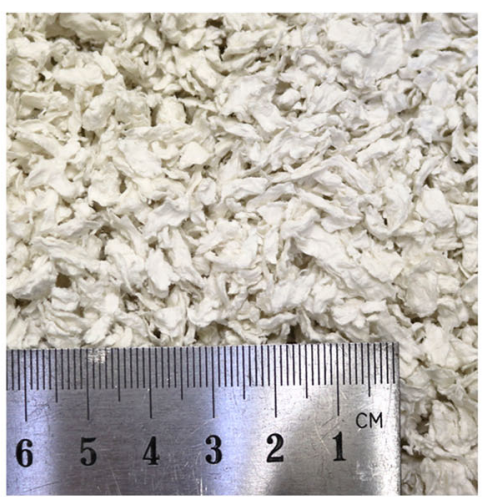

(b)

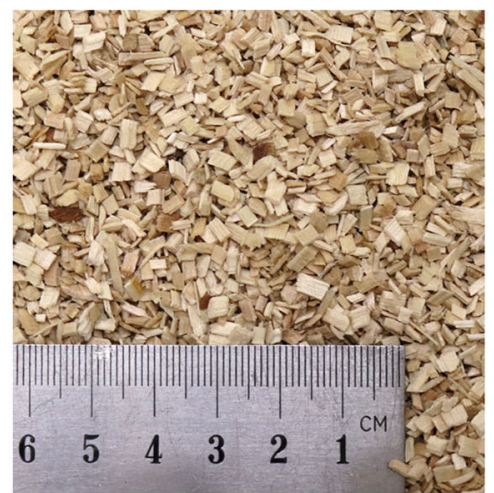

(c)
Fig. 5 Bedding materials used during the experiment. Comfort White (a) and Pure (b) bedding material were compared in the home cagebased preference test and consist of cellulose. c Poplar Granulate bedding material consists of poplar chips. This bedding material was not used in the home cage-based preference test but was used during normal husbandry conditions 
round), only the second day was analyzed, leaving the first for habituation.

\section{Statistical analysis}

During the preference tests, RFID detections by the two RFID antennas were automatically saved by the Arduino onto a microSD card. Each record included a timestamp (synchronized before the start of the experiment via an Internet connection), antenna number (A1 or A2) and the detected RFID tag number. With the help of $\mathrm{R}$ studio (Version 1.1.383, requiring on R 3.0.1+), the data recorded by the Arduino were analyzed for missing detections (see section Data evaluation, Supplements). Following this procedure, cage changes were extracted. In the case of missing detections, in which one RFID antenna did not detect the cage change, the timestamp of the detection of the second antenna was used, arguing that the missing detection resulted from a mouse passing too fast through the tube, which should lead to a roughly similar detection timestamp for both antennas. We decided against subtracting the time spent in the tube from the stay duration. Thus, we calculated stay times for each mouse in each cage as times between cage changes when a mouse entered a new cage (only detections by the antenna passed second).

For each mouse, stay times in each cage were then summed up per day. As already mentioned, we analyzed only the second day of each round because the first day was considered habituation time. Thus, for the investigated $48 \mathrm{~h}$, the percentage of time spent in each cage was calculated for each of the 12 mice. These percentages were then used for further analysis to compare side preference (left vs. right cage) and bedding preference (Pure vs. Comfort White, whereby presentation sides were switched after the first round). To test for normal distribution, the Shapiro-Wilk test was performed in $\mathrm{R}$. The data were considered normally distributed $(p>0.05)$; therefore, a ttest was used to compare the stay time percentages with a chance level of 0.5 (the expected relative stay time if mice had no preference for one of the two cages). In all statistical tests, significance level was set to 0.05 , and result values are given as mean and standard deviation.

\section{Results}

During the two analyzed days, the mice changed cages between 52 and 178 times per $24 \mathrm{~h}(100.75 \pm 31.84$ cage changes). Comparing the times the 12 mice spent in the two cages, we found that during the whole experiment, the mice stayed significantly longer in the right compartment, namely $57.49+/-3.83 \%$ of the time $(\mathrm{t}(11)=-6.77$, p $<0.001$, see Fig. 6a). For the different bedding materials, on the other hand, there was an even clearer preference: the mice stayed $72.76 \pm 3.00 \%$ of the time in the compartment with Comfort White bedding $(\mathrm{t}(11)=-20.19, p<0.001$, see also Fig. 6c).

\section{Discussion}

In this experiment, stay times of the 12 mice on Comfort White and Pure bedding material were compared, whereby stay time was only analyzed after 1 day of habituation, and the presentation side of the bedding was changed inbetween to control for side preference. When looking at

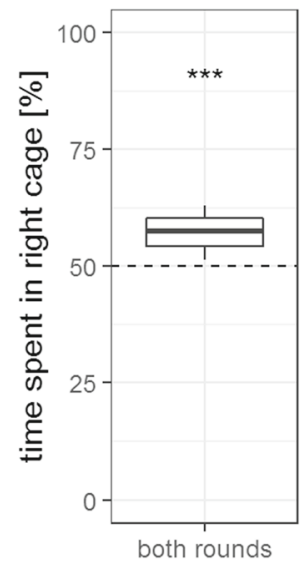

(a)

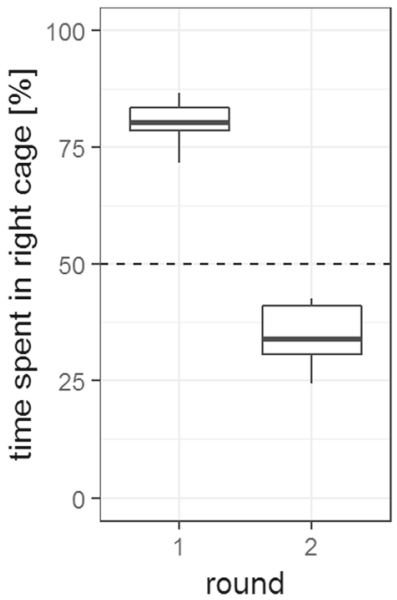

(b)

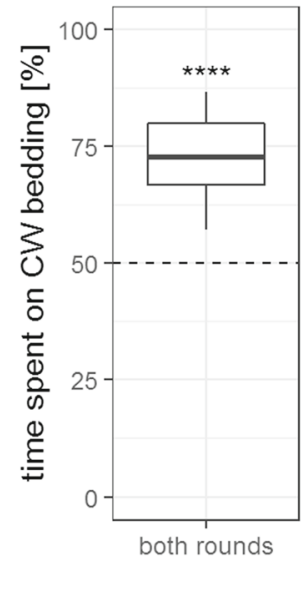

(c)

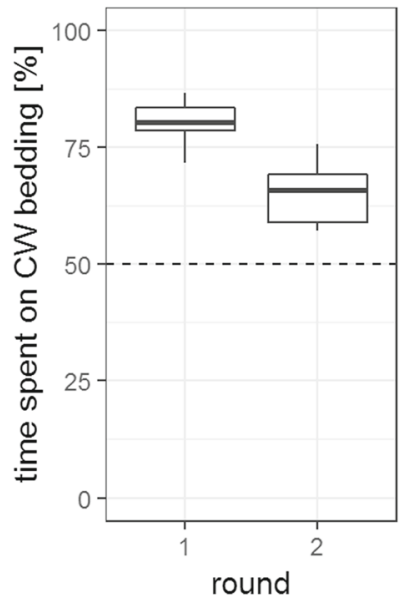

(d)

Fig. 6 Time spent (\%) in the two cages, analyzed by cage side and bedding material. Time spent in the right cage $\mathbf{a}$ in total $(48 \mathrm{~h})$, or $\mathbf{b}$ with regard to round $(24 \mathrm{~h})$. Time spent in the cage with the Comfort White bedding material $\mathbf{c}$ in total $(48 \mathrm{~h}$ ), or $\mathbf{d}$ with regard to round

(24 h). Comfort White bedding material was presented in the right cage during the first round and in the left cage during the second round. $\mathrm{CW}=$ Comfort White $* * * p<1 \times 10^{-4}$, **** $p<1 \times 10^{-9} t$ test comparison to chance level, $n=12$ 
Fig. 6b, which compares the side preference on the second day of both rounds, side preference seems to be more distinct during the first round than the second. This was also reflected in a significant side preference, which could be due to spatial reasons (position in the room etc., Blom et al., 1992). Another explanation could be that the condition preference (for the bedding material) changed over time, becoming less strong and thus leading to a side preference when compared with the round before.

Nevertheless, the mice had a distinct preference for the cage with Comfort White bedding compared to the cage with Pure bedding. Thus, during this home cagebased preference test, we could confirm the results already obtained during the two 10-min observations of the conditioned place preference test: Comfort White bedding is preferred over Pure bedding by this group of 12 C57BL/6J mice.

The main purpose of this experiment was to test the new setup in a week-long experiment as well as to validate the bedding preference previously observed during a conditioned place preference (CPP) test. We have to emphasize that the result of this preference test cannot be generalized for C57BL/6J mice: Although we tested the preference of 12 mice, they were all together as one group in the test system and, thus, might be considered as only one independent sample. Indeed, it is possible that the mice influenced each other in their stay (a) by the behavior of dominant mice, (b) by avoiding or following individual mice, (c) or by preferring to not sleep alone over individual bedding preferences. As stated above, the bedding material was also familiar to the mice and as it was presented first in an experimental environment, it is possible that this might have had an influence. Thus, this test would have to be repeated with more groups with less and younger individuals for a more generalized conclusion. In any case, the preference test was successful in showing the feasibility of the MoPSS even with large group numbers under the experimental conditions of a home cage-based choice test. A study of home cage-based preference tests in which the MoPSS was used for several months to compare different enrichment is currently in preparation.

\section{Conclusions}

In this paper, we offer the construction description to build an automated tracking system that can be used to facilitate the analysis of home cage-based preference test. We showed that the MoPSS is accurate even for fast mice and its error rate can be further reduced close to $0 \%$ with the help of additional logical reconstruction of the data. We also presented an example experiment with the corresponding results in which we compared two different bedding materials.

With this automated tracking system, analysis of home cage-based preference tests will become much easier: They will be less expensive, require less time for the data analysis, and will have much finer data resolution. The MoPSS is able to track individual mice and, therefore, it is suitable for group experiments. In our laboratory, the MoPSS is already being used to compare multiple enrichment conditions with regard to the mice's preference over several months.

We want to emphasize the great advantages of the MoPSS to existing systems: It is even able to detect fast animals and can be easily rebuilt. Currently, we are working on a further improved version with an RFID reader module without proprietary software and increased detection rates. In addition, in the near future, we will be adapting the MoPSS system to be suitable for larger animals such as rats and guinea pigs that require a tube diameter of more than $4 \mathrm{~cm}$. On the basis of the construction description, it is also possible to adjust the MoPSS to other research questions. For example, we are working with a modified MoPSS onto which automated doors and levers or nose poke sensors can be added to test not only for preference but also for the strength of preference by letting the animals work for the access to the other cage (Lewejohann \& Sachser, 2000; Sherwin \& Nicol, 1995, 1996). Using only one RFID antenna, the MoPSS can also be used to record activity data in the home cage. In addition, the MoPSS might also be used to study group dynamics and the influence of individual group members on the position of the whole group.

Supplementary Information The online version contains supplementary material available at https://doi.org/10.3758/s13428-021-01593-7.

Acknowledgements The authors thank the animal caretakers, especially Carola Schwarck, for their support in the animal husbandry.

Funding Open Access funding enabled and organized by Projekt DEAL. This work was funded by the DFG (FOR 2591; LE 2356/5-1). The authors declare no competing interests.

\section{Declarations}

Ethical approval All experiments were approved by the Berlin state authority, Landesamt für Gesundheit und Soziales, under license No. G 0182/17 and were in accordance with the German Animal Protection Law (TierSchG, TierSchVersV).

Publication History This manuscript was previously published on bioRxiv: https://doi.org/10.1101/2020.11.13.379719

Experiments were not preregistered.

Open Access This article is licensed under a Creative Commons Attribution 4.0 International License, which permits use, sharing, adaptation, distribution and reproduction in any medium or format, as long as you give appropriate credit to the original author(s) and the source, provide a link to the Creative Commons licence, and indicate 
if changes were made. The images or other third party material in this article are included in the article's Creative Commons licence, unless indicated otherwise in a credit line to the material. If material is not included in the article's Creative Commons licence and your intended use is not permitted by statutory regulation or exceeds the permitted use, you will need to obtain permission directly from the copyright holder. To view a copy of this licence, visit http://creativecommons. org/licenses/by/4.0/.

\section{References}

Ago, A., Gonda, T., Takechi, M., Takeuchi, T., \& Kawakami, K. (2002). Preferences for paper bedding material of the laboratory mice. Experimental Animals, 51(2), 157-161.

Bains, R. S., Cater, H. L., Sillito, R. R., Chartsias, A., Sneddon, D., Concas, D., ..., Armstrong, J. D. (2016). Analysis of individual mouse activity in group housed animals of different inbred strains using a novel automated home cage analysis system. Frontiers in Behavioral Neuroscience, 10, 106-108.

Baumans, V., Schlingmann, F., Vonck, M., \& van Lith, H. A. H. (2002). Individually ventilated cages: Beneficial for mice and men? Contemporary Topics in Laboratory Animal Science, 41(1), 13-19.

Billat, V. L., Mouisel, E., Roblot, N., \& Melki, J. (2005). Inter- and intrastrain variation in mouse critical running speed. Journal of Applied Physiology, 98(4), 1258-1263.

Blom, H. J. M., Tintelen, G. V., Vorstenbosch, C. J. A. H. V. V., Baumans, V., \& Beynen, A. C. (1996). Preferences of mice and rats for types of bedding material. Laboratory Animals, 30(3), 234-244.

Blom, H., van Vorstenbosch, C., Baumans, V., Hoogervorst, M., Beynen, A., \& van Zutphen, L. (1992). Description and validation of a preference test system to evaluate housing conditions for laboratory mice. Applied Animal Behaviour Science, 35(1), 67-82.

Bono, J. P. D., Adlam, D., Paterson, D. J., \& Channon, K. M. (2006). Novel quantitative phenotypes of exercise training in mouse models. American Journal of Physiology-Regulatory, Integrative and Comparative Physiology, 290(4), R926-R934.

Bridge, E. S., Wilhelm, J., Pandit, M. M., Moreno, A., Curry, C. M., Pearson, T. D., ..., Ruyle, J. E. (2019). An Arduino-based RFID platform for animal research. Frontiers in Ecology and Evolution, 7, 257-267.

Castelhano-Carlos, M., Costa, P. S., Russig, H., \& Sousa, N. (2014). PhenoWorld: A new paradigm to screen rodent behavior. Translational Psychiatry, 4(6), e399-e399.

de Chaumont, F., Coura, R. D.-S., Serreau, P., Cressant, A., Chabout, J., Granon, S., \& Olivo-Marin, J.-C. (2012). Computerized video analysis of social interactions in mice. Nature Methods, 9(4), 410 417.

de Chaumont, F., Ey, E., Torquet, N., Lagache, T., Dallongeville, S., Imbert, A., ..., Olivo-Marin, J.-C. (2019). Real-time analysis of the behaviour of groups of mice via a depth-sensing camera and machine learning. Nature Biomedical Engineering, 3(11), 930942.

de Weerd, H. A. V., Loo, P. L. P. V., Zutphen, L. F. M. V., Koolhaas, J. M., \& Baumans, V. (1997). Preferences for nesting material as environmental enrichment for laboratory mice. Laboratory Animals, 31(2), 133-143.

Endo, N., Ujita, W., Fujiwara, M., Miyauchi, H., Mishima, H., Makino, Y., ..., Kakeyama, M. (2018). Multiple animal positioning system shows that socially-reared mice influence the social proximity of isolation-reared cagemates. Communications Biology 1(1).
Freund, J., Brandmaier, A. M., Lewejohann, L., Kirste, I., Kritzler, M., Kruger, A., ..., Kempermann, G. (2013). Emergence of individuality in genetically identical mice. Science, 340(6133), 756-759.

Freymann, J., Tsai, P.-P., Stelzer, H., \& Hackbarth, H. (2015). The amount of cage bedding preferred by female BALB/c and c57bl/6 mice. Lab Animal, 44(1), 17-22.

Freymann, J., Tsai, P.-P., Stelzer, H., \& Hackbarth, H. (2017). The impact of bedding volumes on laboratory mice. Applied Animal Behaviour Science, 186, 72-79.

Gaskill, B. N., Gordon, C. J., Pajor, E. A., Lucas, J. R., Davis, J. K., \& Garner, J. P. (2012). Heat or insulation: Behavioral titration of mouse preference for warmth or access to a nest. PLOS ONE, 7(3), e32799.

Gaskill, B. N., Rohr, S. A., Pajor, E. A., Lucas, J. R., \& Garner, J. P. (2009). Some like it hot: Mouse temperature preferences in laboratory housing. Applied Animal Behaviour Science, 116(2-4), 279-285.

Gaskill, B. N., Rohr, S. A., Pajor, E. A., Lucas, J. R., \& Garner, J. P. (2011). Working with what you've got: Changes in thermal preference and behavior in mice with or without nesting material. Journal of Thermal Biology, 36(3), 193-199.

Godbey, T., Gray, G., \& Jeffery, D. (2011). Cage-change interval preference in mice. Lab Animal, 40(7), 225-230.

Gordon, C. J., Becker, P., \& Ali, J. S. (1998). Behavioral thermoregulatory responses of single- and group-housed mice. Physiology \& Behavior, 65(2), 255-262. https://doi.org/10.1016/s0031-9384 (98)00148-6. Elsevier.

Gouveia, K., \& Hurst, J. L. (2013). Reducing mouse anxiety during handling: Effect of experience with handling tunnels. PLOS ONE, $8(6)$, e66401.

Habedank, A., Kahnau, P., Diederich, K., \& Lewejohann, L. (2018). Severity assessment from an animal's point of view. Berliner und Münchener Tierärztliche Wochenschrift, 131(7/8), 304-320.

Hollinski, R., Osterberg, A., Polei, S., Lindner, T., Cantré, D., Mittlmeier, T., ..., Müller-Hilke, B. (2018). Young and healthy $\mathrm{c} 57 \mathrm{bl} / 6 \mathrm{j}$ mice performing sprint interval training reveal genderand site-specific changes to the cortical bone. Scientific Reports, 9(1), 1529-39.

Hurst, J. L., \& West, R. S. (2010). Taming anxiety in laboratory mice. Nature Methods, 7(10), 825-826.

Kawakami, K., Shimosaki, S., Tongu, M., Kobayashi, Y., Nabika, T., Nomura, M., \& Yamada, T. (2007). Evaluation of bedding and nesting materials for laboratory mice by preference tests. Experimental Animals, 56(5), 363-368.

Kawakami, K., Xiao, B., ichiro Ohno, R., Ferdaus, M. Z., Tongu, M., Yamada, K., ..., Nabika, T. (2012). Color preferences of laboratory mice for bedding materials: Evaluation using radiotelemetry. Experimental Animals, 61(2), 109-117.

Kirchner, J., Hackbarth, H., Stelzer, H. D., \& Tsai, P.-P. (2012). Preferences of group-housed female mice regarding structure of softwood bedding. Laboratory Animals, 46(2), 95-100.

Kirkden, R. D., \& Pajor, E. A. (2006). Using preference, motivation and aversion tests to ask scientific questions about animals' feelings. Applied Animal Behaviour Science, 100(1-2), 29-47.

Krohn, T. C., \& Hansen, A. K. (2010). Mice prefer draught-free housing. Laboratory Animals, 44(4), 370-372.

Lewejohann, L. (2019a). Severity assessment of experimental procedures by means of conditioned place preference/aversion. Animal Study Registry. https://www.animalstudyregistry.org/10.17590/asr. 0000112, https://doi.org/10.17590/ASR.0000112

Lewejohann, L. (2019b). Severity assessment of experimental procedures by means of conditioned place preference/aversionimproving the procedures. Animal Study Registry. https://www. animalstudyregistry.org/10.17590/asr.0000142, https://doi.org/10. 17590/ASR.0000142 
Lewejohann, L., \& Sachser, N. (2000). Präferenztests zur Beurteilung unterschiedlicher Haltungsbedingungen von männlichen Labormäusen. Aktuelle Arbeiten zur artgemäßen Tierhaltung. KTBL (391), 170-177.

Linnenbrink, M., \& von Merten, S. (2017). No speed dating please! Patterns of social preference in male and female house mice, $14(1), 38-52$.

Loo, P. L. V., Blom, H. J., Meijer, M. K., \& Baumans, V. (2005). Assessment of the use of two commercially available environmental enrichments by laboratory mice by preference testing. Laboratory Animals, 39(1), 58-67.

Loo, P. L. P. V., de Groot, A. C., Zutphen, B. F. M. V., \& Baumans, V. (2001). Do male mice prefer or avoid each other's company? Influence of hierarchy, kinship, and familiarity. Journal of Applied Animal Welfare Science, 4(2), 91-103.

Nath, T., Mathis, A., Chen, A. C., Patel, A., Bethge, M., \& Mathis, M. W. (2019). Using DeepLabCut for 3D markerless pose estimation across species and behaviors. Nature Protocols, 14(7), 2152-2176.

Noldus, L. P. J. J., Spink, A. J., \& Tegelenbosch, R. A. J. (2001). EthoVision: A versatile video tracking system for automation of behavioral experiments. Behavior Research Methods, Instruments, \& Computers, 33(3), 398-414.

Rao, S. R., Olechnowicz, S. W. Z., Krätschmer, P., Jepson, J. E. C., Edwards, C. M., \& Edwards, J. R. (2019). Small animal video tracking for activity and path analysis using a novel open-source multi-platform application (AnimApp). Scientific Reports, 9(1), $12343-12352$.

Redfern, W. S., Tse, K., Grant, C., Keerie, A., Simpson, D. J., Pedersen, J. C., ..., Armstrong, J. D. (2017). Automated recording of home cage activity and temperature of individual rats housed in social groups: The rodent big brother project. PLOS ONE, 12(9), e0s181068.

Shemesh, Y., Sztainberg, Y., Forkosh, O., Shlapobersky, T., Chen, A., \& Schneidman, E. (2013). High-order social interactions in groups of mice. eLife, 2. https://doi.org/10.7554/elife.00759.001. eLife Sciences Publications, Ltd.

Sherwin, C., \& Nicol, C. (1995). Changes in meal patterning by mice measure the cost imposed by natural obstacles. Applied Animal Behaviour Science, 43(4), 291-300.

Sherwin, C., \& Nicol, C. (1996). Reorganization of behaviour in laboratory mice, Mus musculus, with varying cost of access to resources. Animal Behaviour, 51(5), 1087-1093.

Tsai, P. P., Nagelschmidt, N., Kirchner, J., Stelzer, H. D., \& Hackbarth, H. (2012). Validation of an automatic system (DoubleCage) for detecting the location of animals during preference tests. Laboratory Animals, 46(1), 81-84.

Valsecchi, P., \& Galef, B. J. (1989). Social influences on the food preferences of house mice (Mus musculus). International Journal of Comparative Psychology, 2(4), 245-256.

Weissbrod, A., Shapiro, A., Vasserman, G., Edry, L., Dayan, M., Yitzhaky, A., ..., Kimchi, T. (2013). Automated long-term tracking and social behavioural phenotyping of animal colonies within a semi-natural environment. Nature Communications, 4(1), 2018-2028.

Winter, Y., \& Schaefers, A. T. (2011). A sorting system with automated gates permits individual operant experiments with mice from a social home cage. Journal of Neuroscience Methods, 196(2), 276280.

Publisher's note Springer Nature remains neutral with regard to jurisdictional claims in published maps and institutional affiliations. 